\title{
An Intelligent Wireless Sensor Network Temperature Acquisition System with an FPGA
}

\author{
Rachid Souissi ${ }^{1}$, Mohsen Ben-Ammar ${ }^{2}$ \\ ${ }^{1}$ Computer Science Department, Higher Technological Institute of Sfax (ISET), Sfax, Tunisia \\ ${ }^{2}$ Electrical Engineering Department, National Engineering School of Sfax (ENIS) \\ Machine Control and Power Grid Research Unit, CMERP, Sfax, Tunisia \\ Email: rachid.souissi@isetsf.rnu.tn; mohsen.benammar@gmail.com
}

Received September 27, 2013; revised October 27, 2013; accepted November 4, 2013

Copyright (C) 2014 Rachid Souissi, Mohsen Ben-Ammar. This is an open access article distributed under the Creative Commons Attribution License, which permits unrestricted use, distribution, and reproduction in any medium, provided the original work is properly cited. In accordance of the Creative Commons Attribution License all Copyrights (C 2014 are reserved for SCIRP and the owner of the intellectual property Rachid Souissi, Mohsen Ben-Ammar. All Copyright @ 2014 are guarded by law and by SCIRP as a guardian.

\section{ABSTRACT}

The use of FPGAs in the design of wireless sensor networks can improve the processing system performance so as to develop sensor nodes with powerful embedded processor. In fact, FPGA has a super-parallel processing capacity in data processing and its use and configuration is flexible. This allows intensive digital signal processing tasks like Fast Fourier Transforms to be implemented on the sensor node and this can even support intelligent applications and new security algorithms. This paper presents a wireless sensor network system that monitors and visualizes periodically the environment temperature. The proposed system consists of a base station node based on an FPGA DE2-70 and five sensor nodes having the architecture of the System-on-Chip CC2430.

\section{KEYWORDS}

Wireless Sensor Network; ZigBee; System-on-Chip CC2430; FPGA DE2-70

\section{Introduction}

The use of wireless sensor networks (WSNs) has risen in last years. In fact, several industrials, researchers and engineers work on this new technology both in the civilian and military sectors that require the use of many nodes, even hundreds or thousands. That is why more applications have been developed in order to solve several problems in data acquisition and environment control. Moreover, these nodes must be capable of sensing, processing and communicating physical parameters like temperature and pressure through the global wireless network. This paper focuses on the design of a WSN to acquire the temperature from some autonomous nodes. It introduces the use of the FPGA DE2-70 in this application field. The use of the FPGA DE2-70 in WSN can improve the processing system performance so as to develop powerful embedded processor. This paper highlights the different techniques, methods and protocols that can be used in this wireless sensor network technology. The developed system permits monitoring the environment temperature.
The organization of this paper is as follows; In Section 2, we will present some works dealing with sensor nodes mainly based on an FPGA, otherwise Section 3 deals with the IEEE 802.15.4 standard that defines the physical layer in all ZigBee devices; this standard is used by the sensor nodes to communicate with each other. Section 4 is devoted to the system hardware architecture; it exposes the main components of the system. The software architecture is exposed in Section 5. The application description is presented in Section 6. Finally, a conclusion is given to the whole work.

\section{Related Work}

In this section we will present an in-depth knowledge of several wireless sensor networks systems based on FPGA. The reference paper [1] provides an interface and a software/hardware module for Intelligent Car Park Management System based on FPGA. So, in car entering module, as the car enters the lot, it is detected by the IR sensors. The IR sensors provide the pulse to the FPGA which 
assumes that an input is detected and thus the car is entered into the parking lot. As the car leaves the lot, it is detected by the IR sensors. The IR sensors provide the pulse to the FPGA which assumes that an input is detected and thus the car is exited out of the parking lot.

The project [2] has been carried out to develop a novel FPGA-based photosynthesis smart sensor. The smart sensor is capable of acquiring and fusing the primary sensor signals to measure temperature, relative humidity, solar radiation, $\mathrm{CO}_{2}$, air pressure and air flow. This permits the estimation of carbon balance, integrative and derivative variables from net photosynthesis in real time due to the FPGA processing capabilities. To accomplish this, high performance computational capabilities are required. These days, Field Programmable Gate Arrays (FPGAs) are devices that are being employed in applications where high demand computational resources are necessary (Contreras-Medina et al., 2012). Due to the demands for high computation required in this research the use of FPGA-based smart sensor with capabilities of acquiring the primary sensor readings, memory management, signal processing, photosynthesis calculation, wireless communication management and in situ real time photosynthesis signal visualization in a color LCD screen is proposed.

In this work [3], we have developed a modular node. So, both digital and analogue type sensors are being considered. The signals that they produce are conditioned for interfacing to the processing and communications modules. For processing there is a field programmable gate array (FPGA) module. This allows intensive digital signal processing (DSP) tasks such as moving average filters, Fast Fourier Transforms (FFTs) to be implemented on the sensor node and this can even support intelligent applications. The FPGA module was included as this provides a reconfigurable processing solution in which intensive DSP Tasks or in which intelligent functionality can be implemented.

In [4], Wei et al. presented a sensor node based on SoC. Such SoC is based on the OpenRisc1200 processor and executes the $\mu / O S$ II RTOS and Wishbone bus. The SoC consists of three main components: an acquisition unit that is fed from a digital thermometer coupled to the FPGA, the OpenRisc firmware that processes the temperature and a logic block that interfaces a ZigBee transceiver based on RS-232 (SHUNCOM SZ05-STD). The utilized FPGA is an Altera Cyclone II (EP2C70). The system consumes $221 \mathrm{~mW}$. Nevertheless, given that the FPGA only interfaces several components (digital thermometer and ZigBee transmitter), it seems to be an overkill, since its inner embedded resources are not used and the same task can be performed using a low-power microcontroller.

\section{The Communication Stack}

The proposed system uses the IEEE 802.15.4 standard [5] as the communication protocol. The IEEE 802.15.4 standard defines the characteristics of the physical and MAC layers for Low-Rate Wireless Personal Area Networks (LR-WPAN) including wireless sensor networks (WSNs). IEEE 802.15.4 focuses mainly on low-cost, low-power communication between devices and therefore it presents a low transfer rate with a maximum of $250 \mathrm{kbits} / \mathrm{s}$.

\subsection{Physical (PHY) Layer}

The IEEE 802.15.4 standard defines the physical layer (PHY) in all ZigBee devices. The PHY is responsible for data transmission and reception by using a defined radio channel and specific modulation and spreading technique [6]. The IEEE 802.15.4 standard specifies two physical layers that represent three operational frequency bands. These three bands include: $868 \mathrm{MHz}$ (used in Europe), $915 \mathrm{MHz}$ (used in America), and 2.4 GHz (used worldwide) [7]. The 868 and $915 \mathrm{MHz}$ bands are in one PHY using the Binary Phase Shift Keying (BPSK) for modulation, while the $2.4 \mathrm{GHz}$ band is in the second PHY and employs Offset Quadrature Phase Shift Keying (O-QPSK) for modulation. There is a single channel between 868 and $868.8 \mathrm{MHz}, 10$ channels between 902 and $928 \mathrm{MHz}$, and 16 Channels between 2.4 and $2.4835 \mathrm{GHz}$, all using the Direct Sequence Spread Spectrum (DSSS) access mode[6].

\subsection{Medium Access Control (MAC) Layer}

In addition to the physical layer, the IEEE 802.15.4 standard defines the medium access control layer for all ZigBee devices. The MAC layer protocol serves as the interface between the PHY and the higher layer protocols. The functions of the MAC include synchronization, frame validation, acknowledged frame delivery, association, and disassociation [5,8].

The MAC layer defines two types of devices; Full Function Device (FFD) and Reduced Function Device (RFD).

A FFD node implements a full set of MAC layer functions and can communicate with both FFD as well as RFD devices. However, an RFD device implements only a reduced set of functions and it is necessary attached to an FFD node playing the role of a network coordinator (called also PAN coordinator). The PAN coordinator select one of the two operational communication modes defined in IEEE 802.15.4, namely, the non beacon-enabled mode and the beacon-enabled mode, in which beacons are periodically sent by the PAN coordinator to identify and synchronize communication inside its PAN. In both modes, the MAC controls the access to the radio 
channel by employing some methods like the Carrier Sense Multiple Access with Collision Avoidance (CSMA/CA) mechanism. CSMA/CA is a network contention protocol that listens to the network before sending, in order to avoid collision [8].

\subsection{ZigBee Layers}

Based on the IEEE 802.15.4, the ZigBee standard [9] defines the higher layer namely; the network layer and the application layer (Figure 1).

The network layer is responsible for joining/leaving a network, security, routing, discovering 1-hop neighbors and storing neighbor information. The ZigBee network layer supports three topologies; the star topology where end devices (RFD) are attached to a central point playing the role of PAN coordinator (FFD), the tree topology where end devices can be attached also to FFD nodes with routing capabilities playing the role of ZigBee routers in a hierarchical manner (with parent-child relationship), the mesh topology where ZigBee routers can be fully connected.

As shown in Figure 1, the application layer includes the Application Framework, the ZigBee Device Objects (ZDO), and the Application Sub Layer (APS). The Application Framework can have up to 240 Application Objects (APOs), that is, user defined application modules which are part of a ZigBee application [6]. The ZDO defines the role of the device, initiates and responds to binding requests and establishes a secure relationship between devices. The APS offers an interface to data and security services to the APO sand ZDO.

\section{Hardware Architecture}

As shown in Figure 2, our system consists of three types of entities. An end device is a sensor nodes composed by one System-on-Chip (CC2430) and is responsible for sensing environment parameters (such as temperature) and then sending the sensed data to the base station. The base station contains a System-on-Chip (CC2430) and is connected to an FPGA DE2-70 through its expansion header. The base station is responsible for collecting data from end devices. After that, the FPGA DE2-70 reads this information then sends it joined with the address of the appropriate node to the personal computer through one serial link. The PC is responsible for visualizing the received information for further analysis and interpretation [10].

\subsection{The FPGA DE2-70}

The Altera FPGA is widely used as the programmable device to support the Design and Reuse (D \& R). This is even more convenient with the development of System

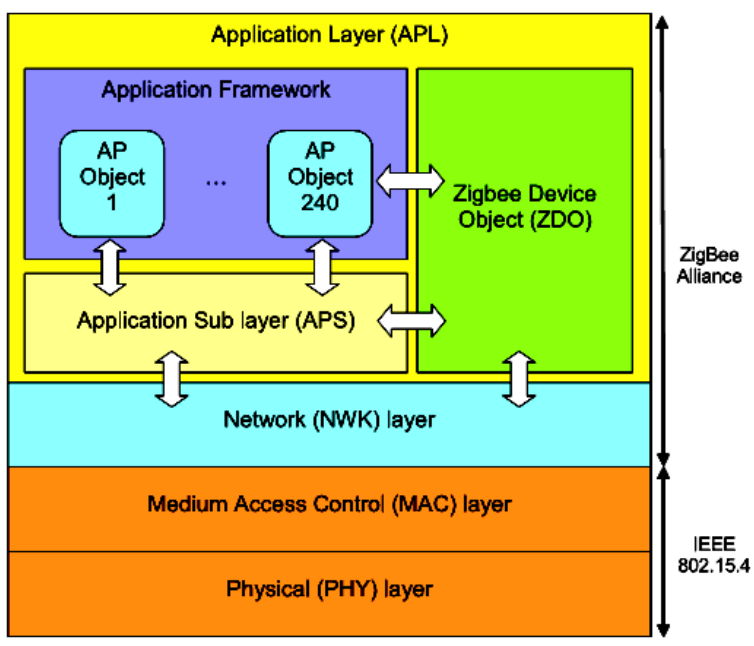

Figure 1. The communication stack.

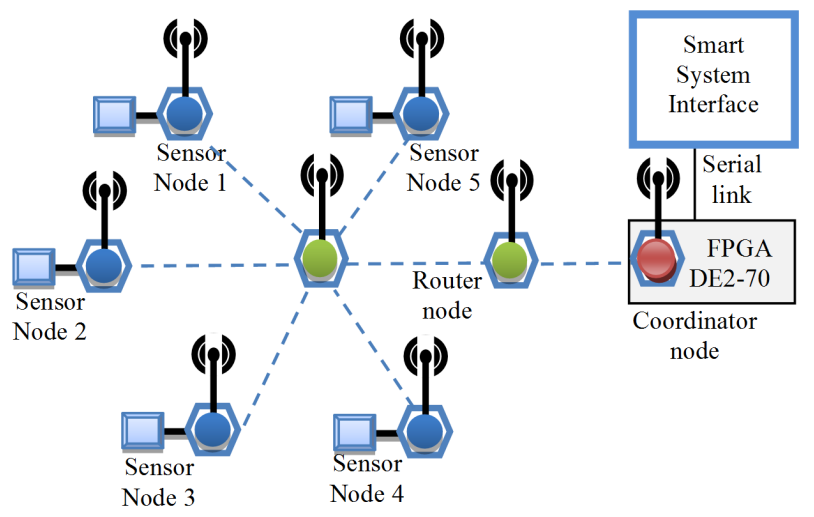

Figure 2. System architecture.

on Programmable Chip (SOPC) where Altera is leading the industry with its FPGA chips and the Quartus Application tools. The Altera Nios is 32-bit softcore RISC processor that is optimized for Altera Field Programmable Gate Array (FPGA) devices. It is a powerful embedded processor and is mainly used to control reconfigurable circuits running on the Altera FPGA. A schema of the DE2-70 board is shown in Figure 3. It depicts the layout of the board and indicates the location of the connectors and key components.

\subsection{BB-Battery Board}

This section describes the System-on-Chip battery board (BB). The main function for this board is to power the CC2430EM (Evaluation Module) or CC2431EM with the use of two AA batteries. It can also be powered by a lab powered connected directly to GND and VDD on the board. The Low-Power RF BB is a simple battery module for use with an EM. As presented in Figure 4, it has one LED, one push switch, one power switch and I/O connector A and B gives access to all $\mathrm{I} / \mathrm{O}$ on the SoC and to some additional pins. It allows you quickly deploy a 


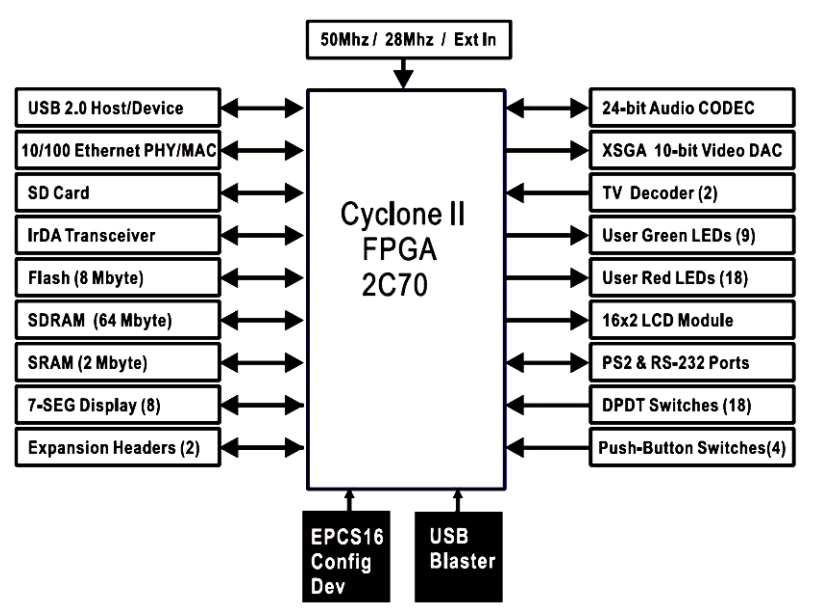

Figure 3. FPGA DE2-70 Board.

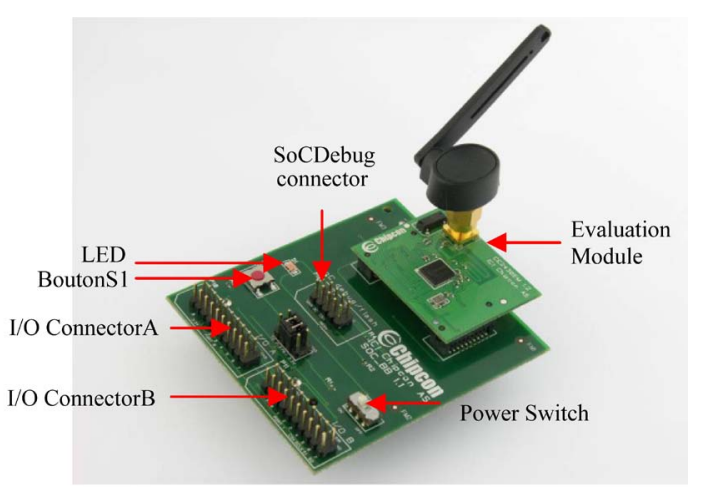

Figure 4. System-on-Chip Battery Board with CC2430EM.

SOC network [11]. The photograph of the component is shown in the Figure 4. On this Battery Board, we can find the Evaluation Module, which contains the minimum Components for a RF part to function.

\subsection{System-on-Chip CC2430}

The CC2430 comes in three different versions: CC2430F32/64/128, with 32/64/128 KB of flash memory respectively. The CC2430 is a true System-on-Chip (SoC) solution specifically tailored for IEEE 802.15.4 and ZigBee $^{\mathrm{TM}}$ applications. It enables ZigBee ${ }^{\mathrm{TM}}$ nodes to be built with very low total bill-of material costs.

The CC2430 combines the excellent performance of the leading CC2420 RF transceiver with an industrystandard enhanced 8051 MCU, 32/64/128 KB flash memory, $8 \mathrm{~KB}$ RAM and many other powerful features. Combined with the industry leading ZigBee ${ }^{\mathrm{TM}}$ protocol stack (Z-Stack), the CC2430 provides the market's most competitive ZigBee ${ }^{\mathrm{TM}}$ solution.

The CC2430 is highly suited for systems where ultralow power consumption is required. This is ensured by various operating modes. Short transition times between operating modes further ensure low power consumption.

\section{4. $8051 \mathrm{CPU}$}

The CC2430 includes an 8-bit CPU core which is an enhanced version of the industry standard 8051 core. The enhanced 8051 core uses the standard 8051 instruction set. Instructions execute faster than the standard 8051 due to the following:

- One clock per instruction cycle is used as opposed to 12 clocks per instruction cycle in the standard 8051;

- Wasted bus states are eliminated. Since an instruction cycle is aligned with memory fetch when possible, most of the single byte instructions are performed in a single clock cycle.

\section{Software Architecture}

The design of the software in this package is based on the layered architecture as depicted in Figure 5.

Application layer: This Software package contains several applications examples with access to Basic RF and HAL (Hardware Abstraction Layer) [12].

Basic RF: This layer offers a simple protocol for transmission and reception on two-way RF link.

Hardware Abstraction Layer: Contains functionality for access to the radio and on-board peripherals modules like LCD, UART, joysticks, buttons, and timers [5].

\subsection{Basic RF}

The Basic RF layer offers a simple protocol for transmission and reception on a two-way RF link. The Basic RF protocol offers the service for packet transmission and reception. It also offers secure communication by use of CCM-64 authentication and encryption/decryption of packets. The security features of Basic RF can be compiled in by defining the compile switch SECURITY_ CCM in the project file. The compile time inclusion of security features is done to save code space for the applications where security features are not needed [5].

The protocol uses IEEE 802.15.4 MAC compliant data and acknowledgment packets. However, it does not offer a full MAC layer, only a simple data link layer for communication between two nodes. Basic RF contains only a small subset of the 802.15.4 standard:

- Association, scanning or beacons are not implemented;

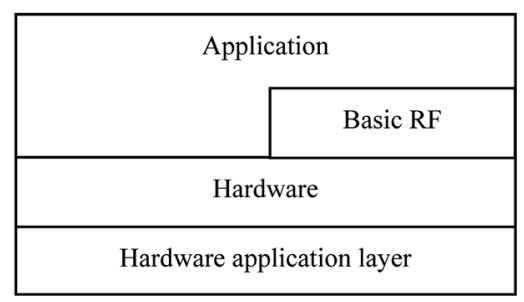

Figure 5. Software architecture. 
- No defined coordinator/device roles (peer-to-peer, all nodes are equal);

- No packet retransmission. This must be taken care of by the layer above Basic RF.

\subsection{Basic RF Instructions}

\subsubsection{Startup}

1) Make sure that the board peripherals and radio interface is initialized i.e. halBoardInit() must have been called first.

2) Create a basicRfCfg_t structure, and initialize its members. If the security features of Basic RF are used, the higher layer is responsible for allocating and assigning the 16 bytes key.

3) Call basicRfInit() to initialize the packet protocol.

\subsubsection{Transmission}

1) Create a buffer with the payload to send. Maximum payload size for Basic RF is 103 Bytes.

2) Call basicRfSendPacket(). Check the return value.

\subsubsection{Reception}

1) Perform polling by calling basicRfPacketIsReady() to check if a new packet is ready to be received by the higher layer.

2) Call basicRfReceive() to receive the packet by higher layer. The caller is responsible for allocating a buffer large enough for the packet and 2 Bytes buffer space for the RSSI value [13].

\subsection{IAR Embedded Workbench ${ }^{\circledR}$ for 8051}

IAR Embedded Workbench is a set of highly sophisticated and easy-to-use development tools for embedded applications. It integrates the IAR $\mathrm{C} / \mathrm{C}++$ Compiler $^{\mathrm{TM}}$, assembler, linker, librarian, text editor, project manager, and C-SPY ${ }^{\circledR}$ Debugger in an integrated development environment (IDE). With its built-in chip-specific code optimizer, IAR Embedded Workbench generates very efficient and reliable FLASH/PROMable code for the 8051 microcontroller. In addition to this solid technology, IAR Systems also provides professional worldwide technical support. So, we have used this environment to write then download program of each node inside his FLASH EPROM.

\section{Results and Discussion}

Our application is composed by two modules "Node()" and "FPGADE270()". The "Node()" moduleis executed on the end devicenodes, while the "FPGADE270()" module is executed on the base stationnode CC2430 (bs) linked to the FPGA DE2-70. The module "Node()" will read the temperature of each node and send the appropri- ate value joined with the address of the node to the main node CC2430 $($ bs $)$ linked to the FPGA DE2-70. When the information is received, it will be sent to the Personal Computer through the serial port where it will be visualised.

\subsection{The "Node" Module}

In this module we have implemented a function that permits to read the temperature. This function will read the temperature of the node, then return the appropriate value to the parent module executed on the node. In fact, in order to get the temperature we have used the predefined functionadcSampleSingle(ADC_REF_1_25_V,ADC_12 BIT,ADC_TEMP_SENS).

\#include <adc.h>

\#ifndef_HAL_TEMP_H

\#define_HAL_TEMP_H

\#define ADC_REF_1_25_V0x00// Internal 1.25V reference

\#define ADC_12_BIT 0x30 // 512 decimation rate

\#define ADC_TEMP_SENS 0x0E// on-chip temperature sensor

\#define CONST 0.61065 // (1250 / 2047)

\#define OFFSET_DATASHEET 743

\#define

OFFSET_MEASURED_AT_25_DEGREES_CELCIUS 29.75

\#define OFFSET (OFFSET_DATASHEET + OFFSET_MEASURED_AT_25_DEGREES_CELCIUS)

// 779.75

\#define TEMP_COEFF 2.45

unsigned char getTemp(void)

\{

unsignedintadcValue;

floatoutputVoltage;

ad-

cValue=adcSampleSingle(ADC_REF_1_25_V,AD

C_12_BIT,ADC_TEMP_SENS);

adcValue $>>=4$

outputVoltage $=$ adcValue $*$ CONST;

return ((outputVoltage - OFFSET) /

TEMP_COEFF); $\}$

When the temperature is acquired, the node will send it to the main node or to his neighbour in general, so we have developed the following module in order to do this RF transmission. So, in the following module we will call the function getTemp() to get the temperature of each node.

\#define PAN_ID 0x2007

\#define CLIENT_ADDR 0x2524

\#define SERVEUR_ADDR 0xBEEF

\#define APP_PAYLOAD_LENGTH 3

static uint8 pTxData[APP_PAYLOAD_LENGTH]; 


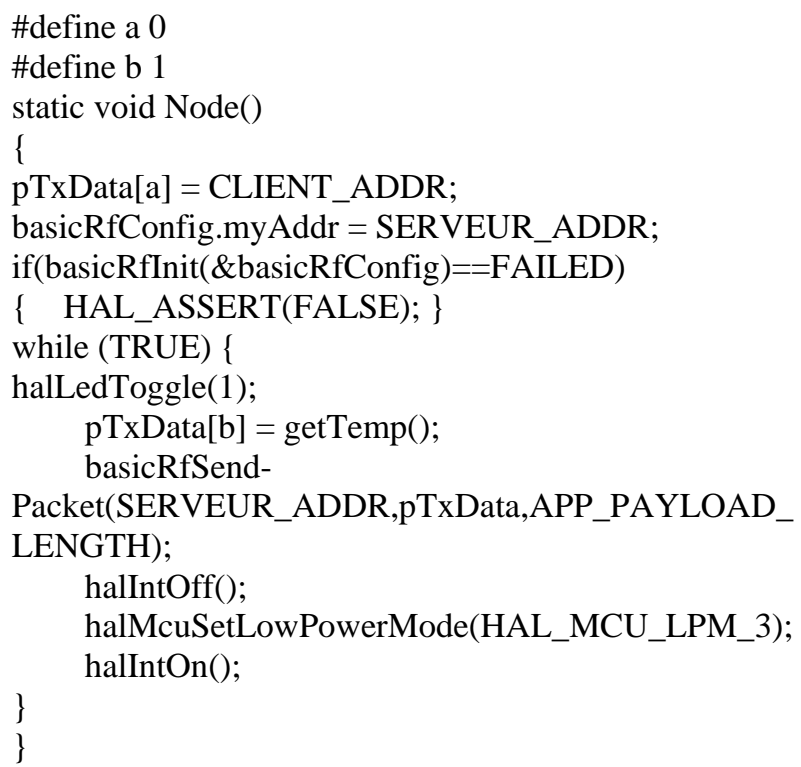

\subsection{The FPGADE270 Module}

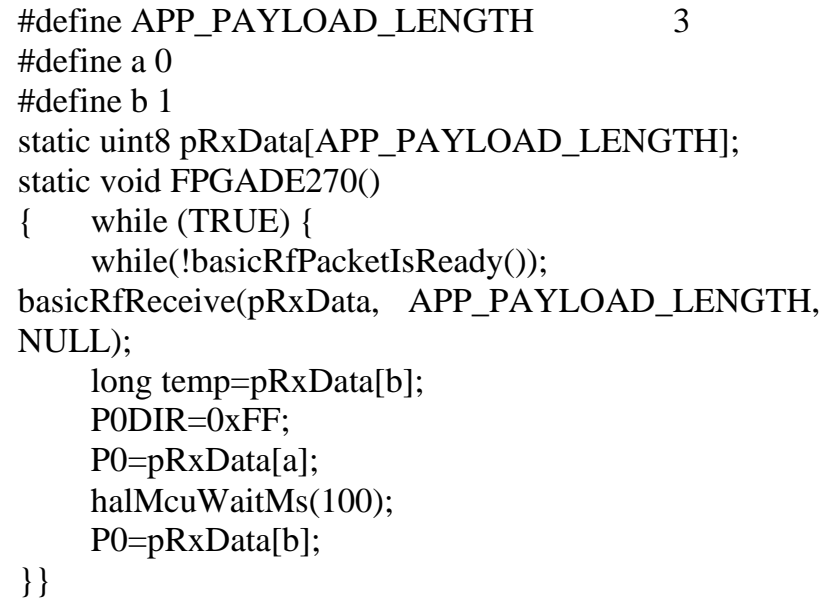

In this module, we receive the appropriate value of the temperature of the node. In fact, to reach this goal we have used the predefined function basicRfReceive(pRx Data, APP_PAYLOAD_LENGTH, NULL) to read the information send by each node. This information is stored in the vector pRxData. So, the temperature is stored in pRxData[b] while the address of the node is in the $\mathrm{pRxData}[\mathrm{a}]$.

\subsection{Monitoring Interface}

When the temperature is received by the FPGADE270 node it will be send later through the serial port to the personal computer where it will be visualised by one interface developed with Matlab Version R2012a as depicted in the following figures. So, the Figure 6(a) illustrates the results taken in hot period like June. Otherwise, in the Figure 6(b) we can see the results related to cold period like December month.

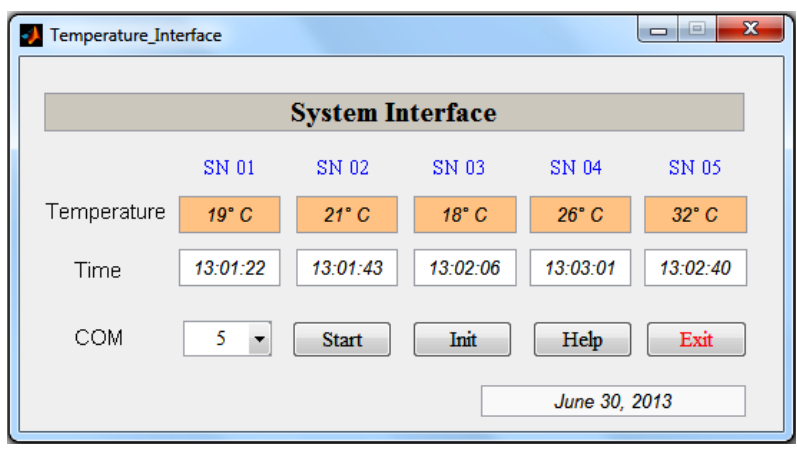

(a)

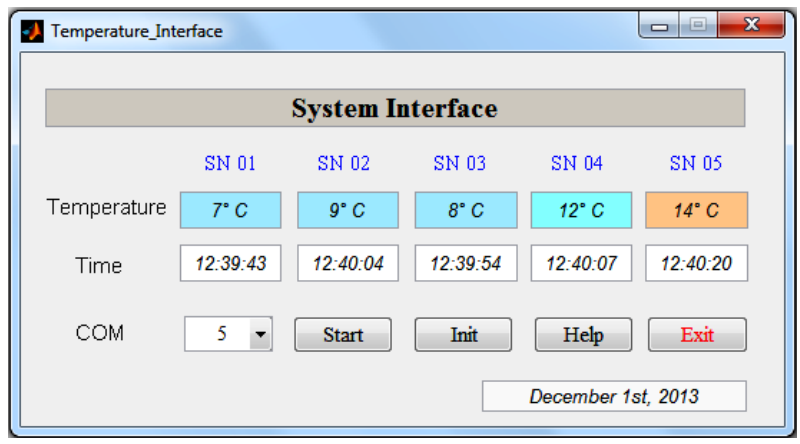

(b)

Figure 6. (a) Acquisition results in June the $30^{\text {th }}$ 2013; (b) Acquisition results in December the $\mathbf{1}^{\text {st }} 2013$.

In this interface we can see an important increase of temperature values send by each node and other information like the time of each received information.

\section{Conclusions}

The development of wireless sensor network reveals new challenges to engineers in several fields. In this context, the development of systems based on wireless sensor networks becomes a necessity. Therefore, actually we are obliged to develop some systems based on this new technology to solve several problems in data acquisition and control in different environments.

Our system includes two aspects: hardware and software. The hardware is composed of one base node CC2430 (bs) connected to the FPGA DE2-70 and five nodes CC2430 ${ }_{(1.5)}$. Each component contains a Systemon-Chip CC2430 that contains the processor which executes the necessary program on each node. The application permits monitoring temperature of the environment. The future work is to develop a synchronisation algorithm to coordinate between transmissions of each node. Moreover, the information received by the WSN will be visualised through the internet network in order to benefit from the distant control and monitoring.

In our system, we have used five nodes to acquire the temperature and send it to the FPGA DE2-70 as a final destination, but if we want to cover a large distance, we 
can use more than five nodes, for example twenty nodes and the distance between two nodes is twenty meter, so each node can send his information to his neighbour node until the final destination or the main node CC2430 $0_{(\mathrm{bs})}$. So, the role of each node is to acquire the temperature from his location, receive and send information to the next node until we reach the last destination. By this way, the WSN will be installed in large area, in order to supervise it.

\section{REFERENCES}

[1] R. Khan, Y. Ali Shah, Z. Khan, K. A. Muhammad and A. M. Amjad Ali, "Intelligent Car Parking Management System on FPGA,” IJCSI International Journal of Computer Science Issues, Vol. 10, No. 1, 2013, pp. 171-173.

[2] J. R. Millan-Almaraz, I. Torres-Pacheco, C. Duarte-Galvan, R. G. Guevara-Gonzalez, L. Miguel Contreras-Medina, R. de Jesus Romero-Troncoso and J. R. Rivera-Guillen, "FPGA-Based Wireless Smart Sensor for Real-Time Photosynthesis," Computers and Electronics in Agriculture, Vol. 93, 2013, pp. 58-69. www.elsevier.com/locate/compag

[3] S. J. Bellis, K. Delaney, B. O’Flynn, J. Barton, K. M. Razeeb and C. O’Mathuna, "Development of Field Programmable Modular Wireless Sensor Network Nodes for Ambient Systems,” University College Cork, Cork, 2005.

[4] J. Wei, L. Wang, F. Wu, Y. Chen and L. Ju, "Design and Implementation of Wireless Sensor Node Based on Open Core," Proceedings of the IEEE Youth Conference on Information, Computing and Telecommunication, Beijing, China, 20-21 September 2009, pp. 102-105.
[5] Institute of Electrical and Electronics Engineers, Inc., IEEE Std.802.15.4-2003, "Wireless Medium Access Control (MAC) and Physical Layer (PHY) Specfications for Low Rate Wireless Personal Area Networks (LRWPANs)," IEEE Press, New York, 2003.

[6] P. Baronti, P. Pillai, V. WC. Chook, S. Chessa, A. Gotta and Y. Fun Hu, "Wireless Sensor Networks: A Survey on the State of the Art and the 802.15.4 and ZigBee Standards," Computer Communications, Vol. 30, No. 7, 2007, pp. 1655-1695.

[7] K. Scott, "Design and Performance of IEEE 802.15.4. Compliant MSE Receivers," Asilomar Conference on Signals, Systems and Computers, Vol. 2, November 2004, pp. 2051-2055.

[8] G. Ding, B. Bhargava, Z. Sahinoglu and P. Zhang, "Reliable Broadcast in ZigBee Networks,” IEEE SECON Proceedings, 2005, pp. 510-520.

[9] ZigBee Alliance, “ZigBee Specifications, ” Version 1.0, 2005.

[10] CC2430 Software Examples Users Guide TEXAS INSTRUMENTS.

[11] Chipcon Products from Texas Instruments, CC2431DK Development Kit User Manual Rev. 1.5.

[12] Y. E. Krasteva, J. Portilla, M. Carnicer, E. de la Torre and T. Riesgo, "Remote HW-SW Reconfigurable Wireless Sensor Nodes,” Industrial Electronics, 2008.

[13] J. Portilla, A. de Castro, E. de la Torre and T. Riesgo, “A Modular Architecture for Nodes in Wireless Sensor Networks,” Journal of Universal Computer Science, Vol. 12, No. 3, 2006, pp. 328-339. 